\section{Nuclear DNA Repair Proteins in Mitochondrial Health and Aging}

\section{Evandro Fei Fang ${ }^{1 *}$, Bradley Wollman ${ }^{1}$, Henok Kassahun ${ }^{1,2,3}$, Hilde Nilsen ${ }^{2,3}$, Morten Scheibye-Knudsen ${ }^{1}$ and Vilhelm A Bohr $^{1 *}$}

'Laboratory of Molecular Gerontology, National Institute on Aging, National Institutes of Health, Baltimore, MD 21224, USA

${ }^{2}$ Institute of Clinical Medicine, Department of Clinical Molecular Biology, University of Oslo, Norway

${ }^{3}$ Section of Clinical Molecular Biology, Akershus University Hospital, Sykehusveien 25, 1474 Nordbyhagen, Norway

As aging occurs, the maintenance of the genome slowly decreases in efficiency [1]. Since the mechanisms of aging are elusive, studies on premature aging diseases provide good models to unveil the mysteries of aging. Cockayne Syndrome/CS (mutation in Csa or Csb), Xeroderma pigmentosum group A (mutation in Xpa), and Ataxia-telangiectasia mutated (mutation in Atm) are segmental premature aging disorders [1]. Each protein is vital in either Double Strand Break repair (DSB), like ATM, or Nucleotide Excision Repair (NER) in CSA, CSB and XPA. Loss of these proteins cause failures in DNA repair and lead to early aging perhaps due to the accumulation of DNA damage [2-4]. The consequences of genomic instability are neurological and developmental issues as well as increased cancer risk among individuals (except in CS) [5].

Among these human premature aging disorders, there is a strong correlation between their phenotypes and mitochondrial health [6]. An imbalance of mitochondrial quality maintenance is associated with mitochondrial diseases and aging. The mitochondrial quality can be maintained by mitochondrial autophagy (termed mitophagy) a cellular process of cleaving damaged mitochondria, and likely facilitates healthy aging [7]. Commonly shared phenotypes between the three diseases are hypersensitivity to exogenous DNA damage inducers (UV light for CS and XP while AT is sensitive to ionizing radiation), shortened lifespan, neurological and cognitive dysfunction such as sensory-neural hearing loss and retinal degeneration $[5,8]$.

*Corresponding author: Evandro F Fang, Laboratory of Molecular Gerontology, National Institute on Aging, National Institutes of Health, Baltimore, MD 21224, USA, Tel: +1 4105588217; E-mail: evandro.fang@nih.gov

Vilhelm A Bohr, Laboratory of Molecular Gerontology, National Institute on Aging, National Institutes of Health, Baltimore, MD 21224, USA, Tel: +1 4105588162 ; E-mail: vbohr@nih.gov

Citation: Fang EF, Wollman B, Kassahun H, Nilsen H, Scheibye-Knudsen M, et al., (2015) Nuclear DNA Repair Proteins in Mitochondrial Health and Aging. J Gerontol Geriatr Med 1: 001.

Received: July 11, 2015; Accepted: July 14, 2015; Published: August 04, 2015
Defective mitophagy seems to contribute to the etiology of mitochondrial dysfunction in these DNA repair deficient-premature aging diseases [2-4]. With the elevated amount of DNA damage, the protein poly [ADP-ribose] polymerase 1 (PARP1) signals the accumulation of DNA repair proteins to repair DNA damage [9]. PARP1 uses large amounts of $\mathrm{NAD}^{+}$which is essential in both PARP1's activity as well as in SIRT1's activity [9]. SIRT1 is an enzyme that is linked to longevity and is involved in the regulation of Uncoupling Proteins (UCPs) that help regulate the mitochondrial membrane potential [9]. Since ATM, CSB and XPA all have phenotypic characteristics of mitochondrial diseases, we supported that the loss of these DNA repair proteins is associated with increased mitochondrial membrane potential, due to a loss of SIRT1 activity, resulting in cleavage of PINK1, a key kinase that regulates mitophagy [9]. PINK1 dysfunction causes defective mitophagy, which promotes altered mitochondrial phenotypes in ATM-, CSB-or XPA-deficient cell lines.

Restoration of autophagy/mitophagy is a promising strategy for healthy aging. A possible intervention is targeting the PARP1 and SIRT1 pathways, both of which need $\mathrm{NAD}^{+}$[10]. It is possible to inhibit PARP1 activity to decrease $\mathrm{NAD}^{+}$utilization allowing more bio available $\mathrm{NAD}^{+}$for SIRT1, but this may increase genomic instability due to PARP1's important role in DNA damage signaling [10]. Nicotinamide Riboside (NR) and Nicotinamide Mononucleotide (NMR) are $\mathrm{NAD}^{+}$precursors which help restore some SIRT1 activity [10]. Increased SIRT1 activity likely contributes to increased longevity in individuals with mitochondrial phenotypes by maintaining mitochondrial health through mitophagy. An increase in healthy mitochondria is beneficial as it should lead to a decrease in ROS production, and decrease unnecessary cell apoptosis [1]. Collectively, these DNA repair deficiency-associated premature aging diseases have mitochondrial dysfunction, and further mechanistic studies of the cause and consequences of these mitochondrial phenotypes as well as the contribution of the mitochondrial dysfunction in the disease progression are of importance.

\section{Disclaimer}

This editorial was written in a personal capacity and does not represent the opinions of the National Institute on Aging, the US Department of Health and Human Services, or the US Federal Government.

\section{Acknowledgements}

The project is supported by the Intramural Research Program of the NIH, National Institute on Aging. We thank Drs. Anne Tseng and Raghavendra Shamanna for critical reading of the manuscript.

\section{Reference}

1. Maynard S, Fang EF, Scheibye-Knudsen M, Croteau DL, Bohr VA (2015) DNA damage, DNA repair, aging, and neurodegeneration. In: Olshansky SJ, Martin GM, Kirldand JL (eds.). Aging: The Longevity Dividend. Cold Spring Harbor Laboratory Press, New York, USA.

2. Shiloh, Y, Ziv Y (2013) The ATM protein kinase: regulating the cellular response to genotoxic stress, and more. Nat Rev Mol Cell Biol 14: 197-210. 
Citation: Fang EF, Wollman B, Kassahun H, Nilsen H, Scheibye-Knudsen M, et al., (2015) Nuclear DNA Repair Proteins in Mitochondrial Health and Aging. J Gerontol Geriatr Med 1: 001

- Page 2 of $2 \cdot$

3. Scheibye-Knudsen, M, Ramamoorthy M, Sykora P, Maynard S, Lin PC, et al. (2012) Cockayne syndrome group B protein prevents the accumulation of damaged mitochondria by promoting mitochondrial autophagy. J Exp Med 209: 855-869.

4. Fang, EF, Scheibye-Knudsen M, Brace LE, Kassahun H, SenGupta T, et al. (2014) Defective mitophagy in XPA via PARP-1 hyperactivation and NAD $(+) /$ SIRT1 reduction. Cell 157: 882-896.

5. Jaarsma D, van der Pluijm, I, van der Horst GT, Hoeijmakers JH (2013) Cockayne syndrome pathogenesis: lessons from mouse models. Mech Ageing Dev 134: 180-195.

6. Scheibye-Knudsen M, Scheibye-Alsing K, Canugovi C, Croteau DL, Bohr VA (2013) A novel diagnostic tool reveals mitochondrial pathology in human diseases and aging. Aging (Albany NY) 5: 192-208
7. Rubinsztein DC, Marino G, Kroemer G (2011) Autophagy and aging. Cell 146: $682-695$

8. Kraemer KH, DiGiovanna JJ (2015) Forty years of research on xeroderma pigmentosum at the US National Institutes of Health. Photochem Photobiol 91: 452-459.

9. Scheibye-Knudsen M, Fang EF, Croteau DL, Bohr VA (2014) Contribution of defective mitophagy to the neurodegeneration in DNA repair-deficient disorders. Autophagy 10: 1468-1469.

10. Guarente $L$ (2014) Linking DNA damage, NAD(+)/SIRT1, and aging. Cell Metab 20: 706-707. 